\title{
Fecal Microbiota Transplantation May Be the Best Option in Treating Multiple Clostridioides difficile Infection: A Network Meta-Analysis
}

\author{
Fanni Dembrovszky (D) · Noémi Gede · Zsolt Szakács (D) • \\ Péter Hegyi (D) · Szabolcs Kiss (D) · Nelli Farkas · Zsolt Molnár (D) • \\ Marcell Imrei · Dóra Dohos (D) · Zoltán Péterfi (iD
}

Received: September 6, 2020 / Accepted: October 8, 2020 / Published online: October 26, 2020

(c) The Author(s) 2020

\begin{abstract}
Introduction: Clostridioides difficile (formerly Clostridium) infection (CDI) is the most common cause of healthcare-associated diarrhea with high mortality and recurrence rate; furthermore, the treatment of recurrent cases is a challenge. In this network meta-analysis, we aimed to compare all available therapies against
\end{abstract}

Electronic Supplementary Material The online version of this article (https://doi.org/10.1007/s40121020-00356-9) contains supplementary material, which is available to authorized users.

F. Dembrovszky · N. Gede · Z. Szakács · P. Hegyi ·

S. Kiss · N. Farkas · Z. Molnár · M. Imrei · D. Dohos

Medical School, Institute for Translational

Medicine, University of Pécs, Pécs, Hungary

Z. Szakács · P. Hegyi

János Szentágothai Research Center, University of

Pécs, Pécs, Hungary

P. Hegyi · S. Kiss · Z. Molnár

Doctoral School of Clinical Medicine, University of

Szeged, Szeged, Hungary

Z. Molnár

Department of Anesthesiology and Intensive

Therapy, Medical Faculty, Poznan University for

Medical Sciences, Poznań, Poland

Z. Péterfi $(\bowtie)$

Division of Infectious Diseases, First Department of Medicine, Medical School, University of Pécs, Pécs, Hungary

e-mail: peterfi.zoltan@pte.hu multiple recurrent CDI (mrCDI) and rank them by efficacy.

Methods: After a systematic search, randomized controlled trials (RCT) with any interventions against mrCDI were included. Data were extracted to the study database using Excel. Risk of bias assessment was performed with the Cochrane RoB 2 tool. The primary outcome was the clinical cure of CDI and the secondary outcome was the recurrence of CDI. A Bayesian method was performed to investigate the efficacy rank order of therapies. We registered our protocol with the Prospero Center for Reviews and Dissemination (registration no. CRD42020160365).

Results: Six RCTs with seven interventions were included in the quantitative synthesis. According to the surface under the cumulative ranking curve values, fecal microbiota transplantation (FMT) after a short course of vancomycin therapy (83\%) shows the highest efficacy for clinical cure. Tolevamer and vancomycin + FMT seemed to be the most effective in preventing recurrence $(87 \%$ and $75 \%$, respectively).

Conclusion: Vancomycin + FMT is perhaps the most effective option for the treatment and prevention of mrCDI, while tolevamer is also effective in preventing recurrence. 
Keywords: CDI; Clostridioides difficile infection; Fecal microbiota transplantation; FMT; Multiple recurrent; Network meta-analysis; Treatment

\section{Key Summary Points}

Many patients infected with Clostridioides difficile experience the recurrence of symptoms after successful treatment.

The treatment of multiple recurrent Clostridioides difficile infection is a challenge worldwide.

Our network meta-analysis advocates that fecal microbiota transplantation after a course of vancomycin therapy seems to be the best treatment option among different interventions tested in randomized controlled trials.

Since fecal microbiota transplantation after vancomycin therapy is effective in reducing symptoms and recurrence rate while being safe, its use can be encouraged in everyday practice.

The limitations of the evidence prompt future randomized controlled trials to validate our findings.

\section{DIGITAL FEATURES}

This article is published with digital features, including a summary slide, to facilitate understanding of the article. To view digital features for this article go to https://doi.org/10.6084/ m9.figshare.13055657.

\section{INTRODUCTION}

Clostridioides difficile (CD) (formerly known as Clostridium difficile) is considered to be the pathogen for one of the most common nosocomial infections. CD is a Gram-positive anerobic spore- and toxin-forming motile bacterium [1], which forms colonies in the colon as part of the normal microbiome in $2-5 \%$ of healthy adults [2].

Clostridioides difficile infection (CDI) is only diagnosed if symptomatic diarrhea occurs (Bristol stool chart types 5-7 more frequently than normally), and its causative role is confirmed by microbiological evidence of fecal CD toxin and toxin-producing CD [3-10]. The symptoms of CDI vary from slight to heavy, with mild cases characterized by watery diarrhea ( $\geq 2-3$ /day), mild abdominal cramping, or tenderness. In severe CDI, patients experience frequent voiding (diarrhea 10-15 times/day) and the scale of symptoms ranges from strong abdominal pain, nausea, and fever to serious local and systemic complications, such as toxic megacolon, pseudomembranous colitis, sepsis, multi-organ failure, and death [11].

CDI can be community-acquired (if the symptoms occur in the community or within $48 \mathrm{~h}$ of admission to a hospital, after no hospitalization in the past 12 weeks) or healthcareacquired (if symptoms occur more than $48 \mathrm{~h}$ after admission or less than 4 weeks after discharge from a healthcare facility or other healthcare-related actions) [12]. The former type is less frequent, since $20-27 \%$ of all CDI cases are community-associated, with an incidence of $20-30$ per 100,000 population $[13,14]$; according to the 2008 survey of European Centre for Disease Prevention and Control conducted in 106 laboratories in 34 European countries, the weighted mean incidence of CDI was 4.1 per 10,000 patient-days per hospital (range 0.0-36.3), and this shows an increasing tendency [15].

The cause of the infection is the disruption of the normal flora in the gastrointestinal tract. This is usually due to previous antibiotic therapy [16], but other healthcare-related actions, such as abdominal surgeries, endoscopic examinations, hospitalization, or living in a sheltered home, are known risk factors. Other important predisposing factors are old age ( $>65$ years), comorbidities, use of certain medications, such as proton-pump inhibitors or nonsteroidal antiinflammatory drugs, chemotherapy, and immunodeficiency [17].

CDI is a potentially life-threatening infection. In addition to its increasing mortality 
(6-30\%) [6, 18] and high recurrence rate (15-30\% after the first episode of CDI, $40 \%$ after the second, and $45-65 \%$ after the third), $[19,20]$ the treatment of CDI is still a major challenge. The first-line recommendations for initial CDI episodes are metronidazole, vancomycin, or fidaxomicin, but, in spite of an initial amelioration of symptoms, a considerable fraction of patients experience relapse $[4,17,21]$. However, it is important to highlight that the Infectious Diseases Society of America recommends both vancomycin and fidaxomicin over metronidazole for initial episodes and does not recommend metronidazole for recurrent CDI (rCDI) [4]. These antibiotics are options after multiple recurrence as well, but other treatments, such as fecal microbiota transplantation (FMT; in combination with antibiotics or alone), probiotics, or even passive immunotherapy with immune whey are available for this indication [17]. Choosing the most successful cure still presents a challenge and imposes an economic burden on the healthcare system so that identification of the most effective treatment option for CDI, especially for multiple recurrent CDI (mrCDI), is a cardinal question.

To rank and compare multiple treatments, a network meta-analysis may be the ideal choice. Although there have been network analyses (NMA) conducted on the efficacy of treatments in CDI [22-27], only one investigated a study population with mrCDI, which was restricted to the efficacy of different FMT methods [22]. Since there are many other therapeutic options available for treating rCDI, expansion of the network to pharmacological therapeutic modalities may provide further evidence when ranking treatments by efficacy. Hence, our aim was to rank and compare the efficacy of all available treatment regimens for mrCDI, thereby contributing to the development of future guidelines.

\section{METHODS}

This NMA was reported according to the Preferred Reporting Items for Systematic Reviews and Meta-Analyses extension statement for interventions (PRISMA-NMA) [28]. We registered our protocol in PROSPERO under registration number CRD42020160365 and fully adhered to it during the study. This article is based on previously conducted studies and does not contain any studies with human participants or animals performed by any of the authors.

\section{Search}

A systematic literature search was performed in four databases, MEDLINE (via PubMed), Embase, Scopus, and Cochrane Central Register of Controlled Trials (CENTRAL), from inception to 29 September 2020, using the following search string: "recurren" AND "clostridium" AND "random*". The search was not limited by any restrictions. In addition, the reference lists in the included studies, previous meta-analyses, and published editorials were screened for eligible studies.

\section{Selection and Eligibility}

Two authors (FD and MI) independently screened titles for eligibility, then abstracts and finally full-text papers. Any disagreement was settled after a discussion with a third author until a consensus was reached. We included randomized controlled trials (RCTs) and used the $\mathrm{PICO}(\mathrm{ST})$ framework to form our clinical question: $\mathrm{P}$ (population)-adult patients with mrCDI (more than one recurrence); I (intervention), $\mathrm{C}$ (comparison)-any interventions used to treat CDI; O (outcome)-clinical cure and recurrence of the infection (recurrence is defined as the reoccurrence of CDI within 8 weeks or within the follow-up period in the case of RCTs analyzed after the onset of a previous episode, provided the symptoms from the previous episode were resolved after completing initial treatment of CDI-related symptoms) [6, 29]; S (study type)-RCT; T (timing)6-10 weeks.

Patients had to be adults (aged at least 18 years) with confirmed CDI (defined as diarrhea plus a positive $\mathrm{CD}$ nucleic acid amplification test, positive cytotoxin assay result, or pseudomembranous colitis). Studies of patients 
with primary or non-multiple recurrence of CDI (which would have a different prognosis from the overall patient cohort with CDI) were excluded from the analysis. The studies also had to report the symptomatic cure.

The primary outcome of our NMA was sustained clinical cure, which was defined as the number of patients cured (defined as the resolution of diarrhea, as defined by individual trial criteria at the end of the follow-up period). The secondary outcome was the recurrence of CDIassociated diarrhea within the follow-up period.

\section{Data Extraction and Analysis}

The extraction was independently conducted by two authors (FD and MI) in duplicate, with any disagreement settled after discussion with a third author until consensus was reached. A predesigned Excel spreadsheet (Microsoft Corporation, Redmond, WA, USA) was used to extract the following data: general details of the study (authors, year of publication, and study site), participants' information (sample size, age, and gender), the characteristics of the treatments, and the main outcome measures according to per protocol data. Only data published in the original articles were extracted; no supplementary information was obtained. There was no overlapping population or duplicate data.

A Bayesian method was used to perform pairwise meta-analyses and a network metaanalysis with the random effect model. Risk ratios (RR) were calculated for dichotomous data with 95\% credible intervals (95\% CrI). We optimized the model and generated posterior samples using four-chain Markov chain Monte Carlo methods. We set at least 20,000 adaptation iterations to achieve convergence and 10,000 simulation iterations. Network estimates (pooled direct and indirect data) of each intervention compared to standard medical therapy and to other interventions are presented in forest plots, summarized in a league table. We also ranked interventions by their posterior probability by calculating the surface under the cumulative ranking (SUCRA) curve values. Funnel plots were created for both outcomes, and Egger's tests were performed to assess the small-study effect. All calculations were performed with the gemtc package (V. 0.8-2) in R (V. 3.5.2) along with the Markov chain Monte Carlo engine JAGS (V. 3.4.0), the netmeta package (V. 1.1-0), and STATA 16.0 (StataCorp LLC).

\section{Risk of Bias Assessment and Certainty of Evidence}

Version 2 of the Cochrane risk of bias tool (RoB 2) [30], which is dedicated to assessing RCTs, was used by two authors to appraise risk of bias (RoB) in each of the included studies. Any disagreement was settled after a discussion with a third author until a consensus was reached. The assessment was first completed on the individual study level. Then we chose the one individual study which showed the highest risk of bias, and we summarized the overall RoB assessment of the interventions on the comparison level with the same method. The Grading of Recommendations Assessment, Development and Evaluation Working Group modality (GRADE) approach was used to assess certainty of evidence for both outcomes for each pairwise comparison [31]. A grading process was independently used by two authors (FD and $\mathrm{MI})$, and disagreements were resolved by a third author. A detailed description of the quality assessment process can be found in the Supplementary Material.

\section{RESULTS}

\section{Systematic Search and Selection}

The process of the systematic search and study selection is illustrated in Fig. 1. After careful selection, six RCTs [32-37] were eligible for the quantitative synthesis.

\section{Study Characteristics}

The characteristics of the included articles are presented in Table 1. The studies reported on 310 patients with mrCDI, were published 
between 2008 and 2019, and investigated seven different interventions for mrCDI. The followup period ranged between 6 and 10 weeks. All included trials had an active comparator (three two-arm and three three-arm trials). All studies reported on both outcomes. The geometry of the network is shown in Fig. $2 \mathrm{a}$.

\section{Ranking and Pairwise Comparisons}

An analysis which compares the resolution of CDI symptoms after completing certain therapies found no statistically significant difference between the interventions. However, FMT after vancomycin pretreatment showed the best SUCRA probability (83\%) (see Fig. $2 \mathrm{~b}$ and Supplementary Fig. 2A), while the SUCRA values of the most frequently used antibiotics were lower (fidaxomicin, 60\%; metronidazole, 51\%; vancomycin, 47\%). Therefore, a combination of vancomycin and FMT seems to be the most effective option for treating mrCDI. In the case of our secondary outcome, recurrence of symptoms, tolevamer and vancomycin + FMT were ranked first and second (with SUCRAs of $87 \%$ and $75 \%$, respectively) compared to the other interventions (51-32\%). The pairwise comparisons of the interventions are shown in Fig. 2b for primary outcome and in the league table in Supplementary Fig. S1 for secondary outcome.
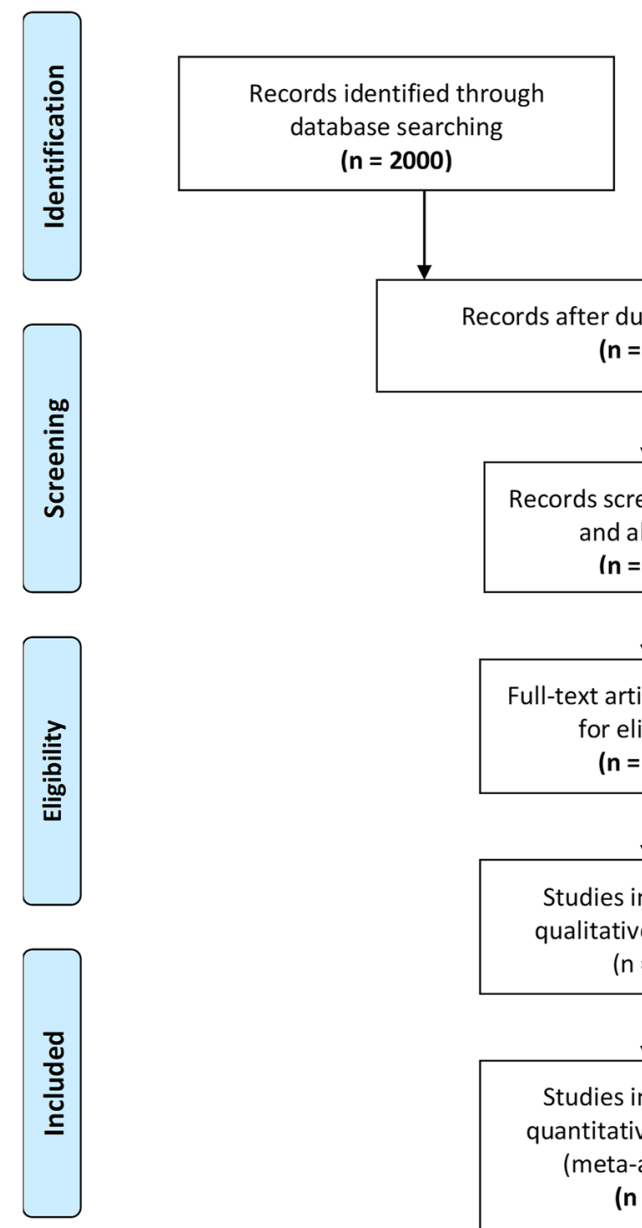

Additional records identified through other sources ( $n=2000)$

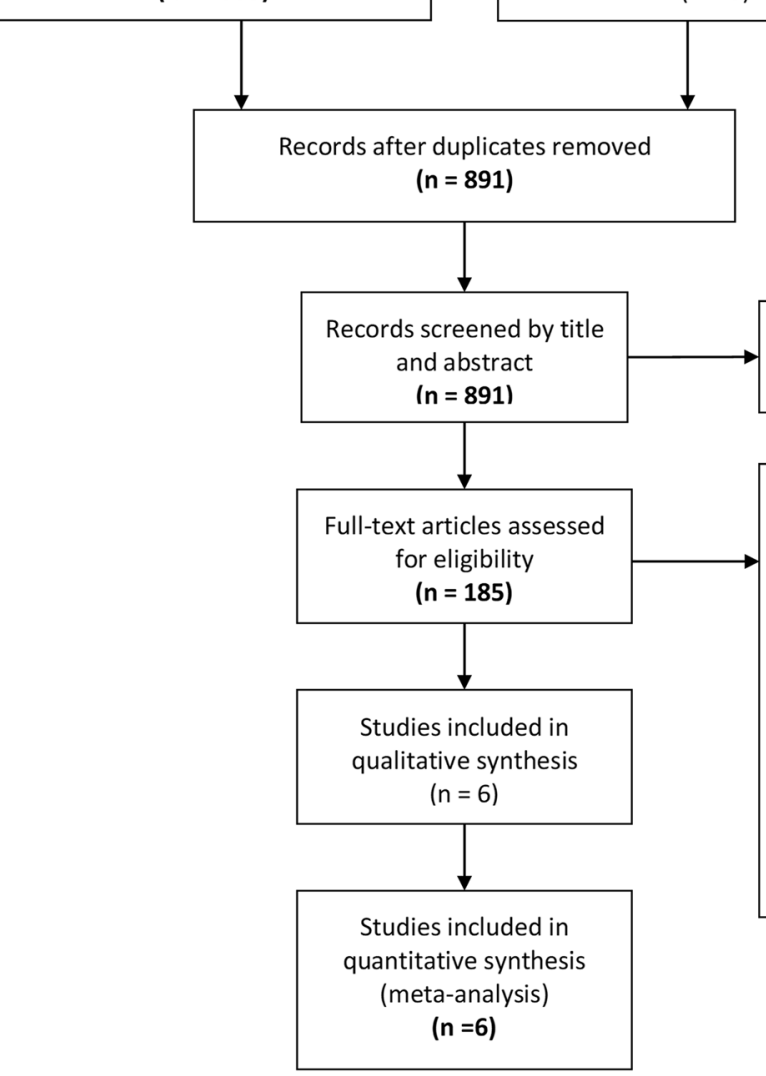
$(n=0)$

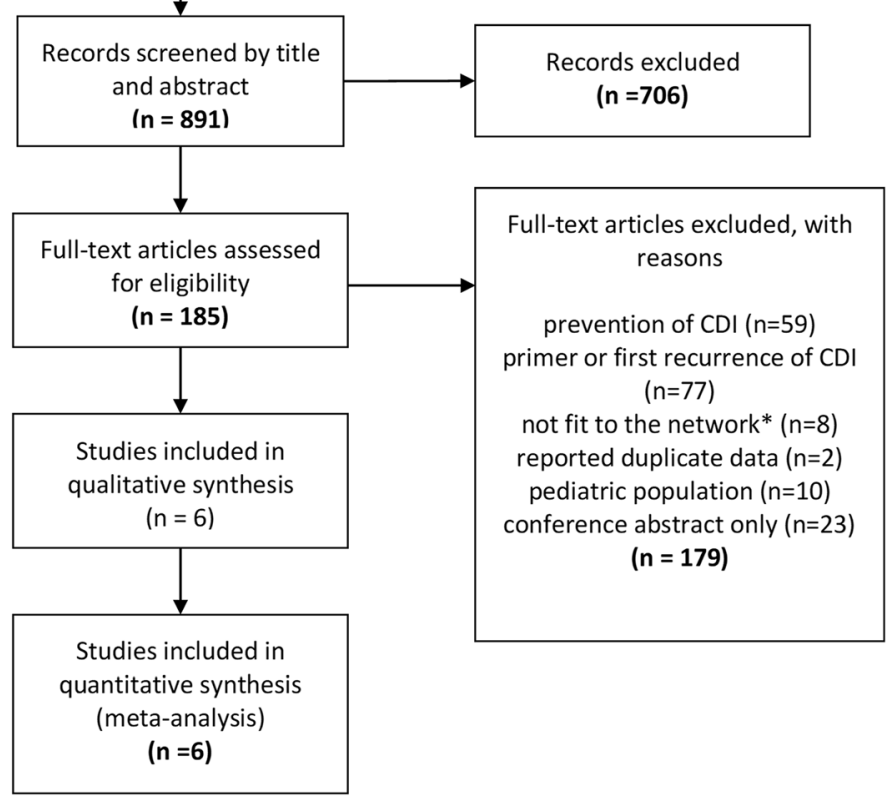

Fig. 1 Study selection process. PRISMA flowchart containing results of systematic search and article selection. ${ }^{*}$ RCTs comparing different FMT methods cannot be connected to our network. CDI Clostridioides difficile infection 


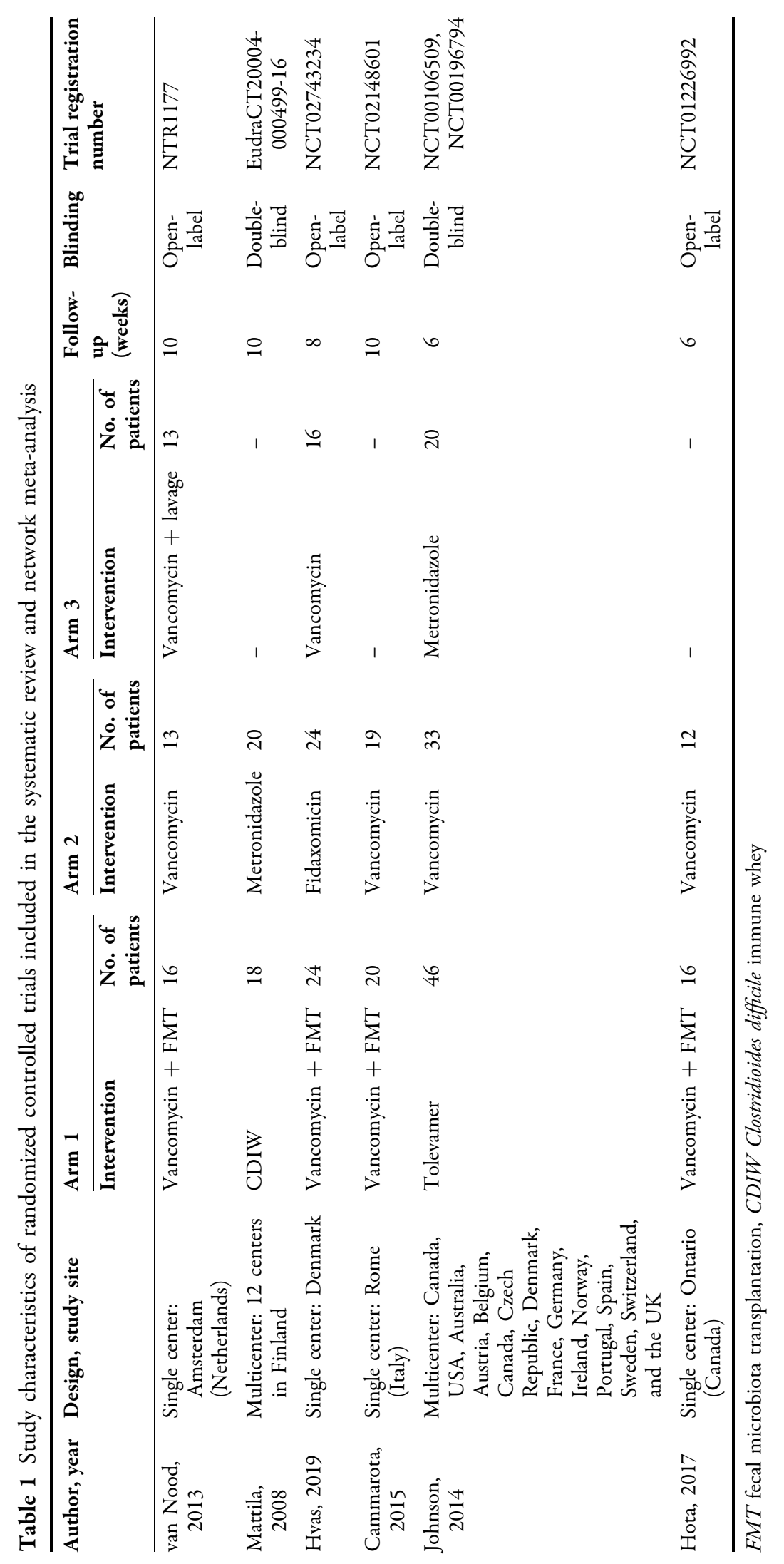


a

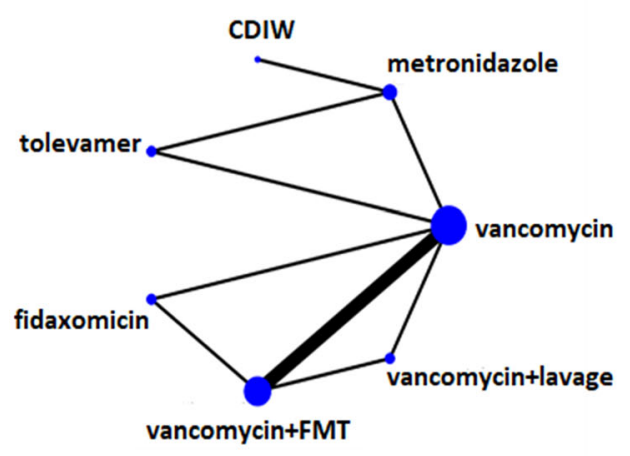

C

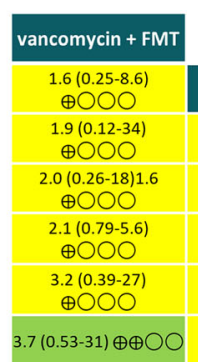

\section{fidaxomicin}

$1.2(0.055-36)$ $\oplus 000$ $1.3(0, .10-18)$ $\oplus \mathrm{OOO}$

$1.3(0.24-8.7)$

$\oplus 000$

$2.0(0.16-33)$

$\oplus \mathrm{O} 0 \mathrm{O}$

$2.4(0.19-37)$

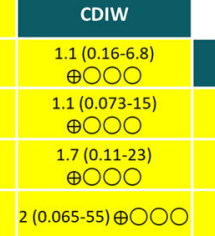

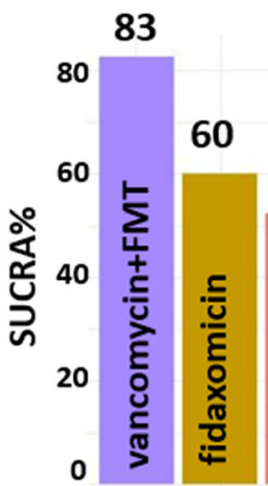
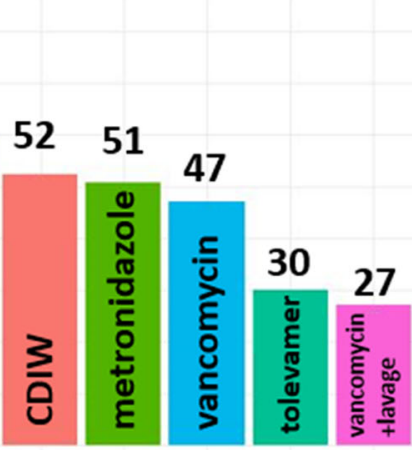

Treatments

Fig. 2 Results of the primary outcome. a Network map for interventions against mrCDI included in the six RCT studies. The size of the nodes on this map reflects the number of included studies for each treatment, whereas the edge thickness is proportional to the number of head-tohead trials. b Ranking by surface under the cumulative ranking curves (SUCRA\%) values of the primary outcome. The length of the columns is proportional to the SUCRA values. c League table for pairwise comparisons. (The comparison is always between the upper and lower

\section{Risk of Bias Assessment and Quality of Evidence}

The overall risk of bias of the head-to-head comparisons for our primary outcome are shown in Fig. 2c, while Supplementary Fig. S1 shows the secondary outcome. Most of the pairwise comparisons raise some problems because only two studies used blinding and only one study has a statistical analysis plan. Two pairwise comparisons have a low risk of bias for each outcome. We do not report any b

metronidazol

$1.0(0.16-8.8)$ $\oplus \mathrm{OOO}$

$1.6(0.25-9.8)$ $\oplus 000$ $1.9(0.12-32)$
$\oplus 000$

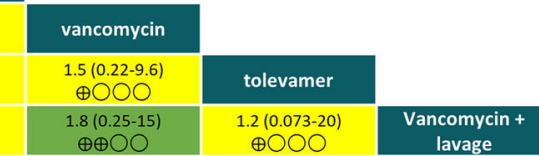

column.) Values are given as relative risk (95\% credibility interval). The colors of the boxes represent the overall RoB assessment of the comparisons (green, low risk of bias; yellow, some concerns). The number of $\oplus$ symbols points to the low level of evidence based on the GRADE approach $(\oplus \oplus \bigcirc)$, low level of evidence; $\oplus \bigcirc \bigcirc \bigcirc$, very low level of evidence). Abbreviations: CDIW: Clostridioides difficile immune whey, FMT: fecal microbiota transplantation

publication bias, since a visual assessment and the results of Egger's test do not suggest that it is present. The risk of bias assessment of the included studies is presented in Supplementary Fig. S3A and B.

The quality of evidence in the studies was variable (see Fig. 2c, Supplementary Fig. S1, Table S1). The grade of evidence was of low or very low quality for each pairwise comparison between interventions. 


\section{DISCUSSION}

The present NMA was conducted to compare the different treatments for mrCDI. Our aim was to assemble a rank order of the interventions and determine the most effective one in treating this infection and in preventing a further recurrence. Our NMA of RCTs showed that FMT after vancomycin therapy is the most effective cure, and it is the second option in effectiveness as regards recurrence. Although tolevamer ranked the highest for recurrence, it must be noted that it is no longer used in routine clinical practice. One of the main reasons for its withdrawal from daily clinical practice may be side effects, such as intestinal disturbances, including loss of appetite, nausea, vomiting, and constipation. Changes in blood electrolytes, such as hypomagnesemia, hypocalcemia, and hypokalemia, could also contribute to unwanted effects.

To the best of our knowledge, this study is the first NMA to investigate the effectiveness of all the treatments (not only FMT) against multiple recurrent cases of CDI included in the published RCTs. In Rokkas et al.'s previous NMA comparing different modalities of FMT with certain antibiotics, donor FMT showed the highest efficacy in comparison with other therapeutic options [22]. It is important to highlight that they only analyzed RCTs which compared FMT with other therapeutic interventions against rCDI, while we included RCTs with any intervention. In contrast with our results on mrCDI, one NMA which analyzed 13 different pharmacological interventions in a population of first and second CDI found that fidaxomicin and teicoplanin were the most effective [24]. FMT was not included to this analysis, since it is not recommended for primary infection. Other NMAs only investigated the efficacy of determined interventions (FMT, bezlotoxumab, only probiotics, only antibiotics or other antimicrobial agents [23, 25-27]), so our NMA can provide a more comprehensive result.

The current guidelines recommend vancomycin or fidaxomicin for non-severe mrCDI cases with the same effectiveness $[4,17]$ or vancomycin in the first line [21]. However, as a result of the release date of the latest European guideline, FMT is only mentioned as an option for multiple recurrent cases, since the first RCT on FMT was published in 2013. On the other hand, the latest Infectious Diseases Society of America (IDSA) and Society for Healthcare Epidemiology of America (SHEA) guidelines only advocate the use of FMT for mrCDI after a failed appropriate antibiotic treatment [4]. Furthermore, in its updated guidelines, the Australasian Society of Infectious Diseases recommends the use of FMT following the failure of less invasive options and claims that there are no data comparing the acceptability and effectiveness of FMT, fidaxomicin, and orally administered vancomycin for multiple CDI recurrences [21]. However, the Australian consensus statement for the use of FMT recommends it for recurrent CDI cases with a high quality of evidence [38]. Considering how recently the RCTs were analyzed, our findings may provide additional information for the current guidelines.

The aim of FMT is to reconstitute normal flora by transplanting healthy donor feces. In addition to its efficacy, FMT appears to be a safe intervention with mild to moderate adverse events, such as abdominal discomfort and nausea, but they are generally self-limited $[33,34]$. It is important to note that failure to preserve the normal gastrointestinal flora is a factor in severe, recurrent, and prolonged CDI cases [39].

Despite the positive message on the efficacy of FMT in mrCDI, the current NMA has some limitations. Risk of bias in some of the RCTs cannot be ruled out. For example, more than half of the studies did not report if participants were blinded to the intervention. Because of this, most of the studies using this analysis were graded as being of very low or low quality. Most of them did not publish a pre-specified statistical analysis plan, hence suggesting an increased risk of bias. Another major limitation is that almost all the trials included a small sample of patients. Furthermore, different FMT protocols were used, including variable FMT routes and preparations, antibiotic dosage, and follow-up time. 


\section{CONCLUSION}

The results of our NMA show that FMT after vancomycin pretreatment has a high success rate for treating mrCDI. This finding is expected to have an influence on therapeutic guidelines in the future and to help make FMT a highly recommended therapy for mrCDI.

However, there are still some unanswered questions. Well-designed RCTs with bigger study populations, well-defined interventions, and longer follow-up periods are needed to ensure the effectiveness and safety of FMT.

\section{ACKNOWLEDGEMENTS}

Funding. The article was funded by the GINOP-2.3.2-15-2016-00048-STAY ALIVE project co-financed by the European Union (European Regional Development Fund) within the framework of the Széchenyi 2020 program and a Human Resources Development Operational Program Grant, Grant Number: EFOP-3.6.2-162017-00006-LIVE LONGER co-financed by the European Union (European Regional Development Fund) within the framework of the Széchenyi 2020 program. The Rapid Service Fee was funded by the authors.

Authorship. All named authors meet the International Committee of Medical Journal Editors (ICMJE) criteria for authorship for this article, take responsibility for the integrity of the work as a whole, and have given their approval for this version to be published.

Disclosures. Fanni Dembrovszky, Noémi Gede, Zsolt Szakács, Péter Hegyi, Szabolcs Kiss, Nelli Farkas, Zsolt Molnár, Marcell Imrei, Dóra Dohos and Zoltán Péterfi declare that they have no conflict of interest with respect to the research, authorship and/or publication of this article.

Compliance with Ethic Guidelines. This article is based on previously conducted studies and does not contain any studies with human participants or animals performed by any of the authors.

Data Availability. The data that support the findings in this study are available from the corresponding author upon reasonable request.

Open Access. This article is licensed under a Creative Commons Attribution-NonCommercial 4.0 International License, which permits any non-commercial use, sharing, adaptation, distribution and reproduction in any medium or format, as long as you give appropriate credit to the original author(s) and the source, provide a link to the Creative Commons licence, and indicate if changes were made. The images or other third party material in this article are included in the article's Creative Commons licence, unless indicated otherwise in a credit line to the material. If material is not included in the article's Creative Commons licence and your intended use is not permitted by statutory regulation or exceeds the permitted use, you will need to obtain permission directly from the copyright holder. To view a copy of this licence, visit http://creativecommons.org/licenses/by$\mathrm{nc} / 4.0 /$.

\section{REFERENCES}

1. Carroll KC, Bartlett JG. Biology of Clostridium difficile: implications for epidemiology and diagnosis. Annu Rev Microbiol. 2011;65:501-21.

2. Schäffler H, Breitrück A. Clostridium difficile - from colonization to infection. Front Microbiol. 2018;9: 646.

3. Bauer MP, Kuijper EJ, Van Dissel JT, European Society of Clinical Microbiology and Infectious Diseases. European Society of Clinical Microbiology and Infectious Diseases (ESCMID): treatment guidance document for Clostridium difficile infection (CDI). Clin Microbiol Infect. 2009;15(12):1067-79.

4. McDonald LC, Gerding DN, Johnson S, et al. Clinical Practice Guidelines for Clostridium difficile infection in adults and children: 2017 update by the Infectious Diseases Society of America (IDSA) and Society for Healthcare Epidemiology of America (SHEA). Clin Infect Dis. 2018;66(7):e1-48. 
5. Bartlett JG, Gerding DN. Clinical recognition and diagnosis of Clostridium difficile infection. Clin Infect Dis. 2008;46(Suppl 1):S12-8.

6. Kuijper EJ, Coignard B, Tüll P. Emergence of Clostridium difficile-associated disease in North America and Europe. Clin Microbiol Infect. 2006;12(Suppl 6):2-18.

7. Crobach MJ, Dekkers OM, Wilcox MH, Kuijper EJ. European Society of Clinical Microbiology and Infectious Diseases (ESCMID): data review and recommendations for diagnosing Clostridium difficileinfection (CDI). Clin Microbiol Infect. 2009;15(12): 1053-66.

8. Lewis SJ, Heaton KW. Stool form scale as a useful guide to intestinal transit time. Scand J Gastroenterol. 1997;32(9):920-4.

9. McDonald LC, Coignard B, Dubberke E, et al. Recommendations for surveillance of Clostridium difficile-associated disease. Infect Control Hosp Epidemiol. 2007;28(2):140-5.

10. O'Donnell LJ, Virjee J, Heaton KW. Detection of pseudodiarrhoea by simple clinical assessment of intestinal transit rate. BMJ. 1990;300(6722):439-40.

11. Knoop FC, Owens M, Crocker IC. Clostridium difficile: clinical disease and diagnosis. Clin Microbiol Rev. 1993;6(3):251-65.

12. Gupta A, Khanna S. Community-acquired Clostridium difficile infection: an increasing public health threat. Infect Drug Resist. 2014;7:63-72.

13. Centers for Disease Control and Prevention (CDC). Severe Clostridium difficile-associated disease in populations previously at low risk-four states, 2005. MMWR Morb Mortal Wkly Rep. 2005;54(47): 1201-1205.

14. Wilcox MH, Mooney L, Bendall R, Settle CD, Fawley WN. A case-control study of community-associated Clostridium difficile infection. J Antimicrob Chemother. 2008;62(2):388-96.

15. Bauer MP, Notermans DW, van Benthem BHB, et al. Clostridium difficile infection in Europe: a hospitalbased survey. Lancet. 2011;377(9759):63-73.

16. Freedberg DE, Salmasian H, Cohen B, Abrams JA, Larson EL. Receipt of antibiotics in hospitalized patients and risk for Clostridium difficile infection in subsequent patients who occupy the same bed. JAMA Intern Med. 2016;176(12):1801-8.

17. Debast SB, Bauer MP, Kuijper EJ. European Society of Clinical Microbiology and Infectious Diseases: update of the treatment guidance document for
Clostridium difficile infection. Clin Microbiol Infect. 2014;20(Suppl 2):1-26.

18. Cambridge B, Cina SJ. The accuracy of death certificate completion in a suburban community. Am J Forensic Med Pathol. 2010;31(3):232-5.

19. Doh YS, Kim YS, Jung HJ, et al. Long-term clinical outcome of Clostridium difficile infection in hospitalized patients: a single center study. Intest Res. 2014;12(4):299-305.

20. Marsh JW, Arora R, Schlackman JL, Shutt KA, Curry SR, Harrison LH. Association of relapse of Clostridium difficile disease with BI/NAP1/027. J Clin Microbiol. 2012;50(12):4078.

21. Trubiano JA, Cheng AC, Korman TM, et al. Australasian Society of Infectious Diseases updated guidelines for the management of Clostridium difficile infection in adults and children in Australia and New Zealand. Intern Med J. 2016;46(4):479-93.

22. Rokkas T, Gisbert JP, Gasbarrini A, et al. A network meta-analysis of randomized controlled trials exploring the role of fecal microbiota transplantation in recurrent Clostridium difficile infection. United Eur Gastroenterol J. 2019;7(8):1051-63.

23. Alhifany AA, Almutairi AR, Almangour TA, et al. Comparing the efficacy and safety of faecal microbiota transplantation with bezlotoxumab in reducing the risk of recurrent Clostridium difficile infections: a systematic review and Bayesian network meta-analysis of randomised controlled trials. BMJ Open. 2019;9(11):e031145.

24. Beinortas T, Burr NE, Wilcox MH, Subramanian V. Comparative efficacy of treatments for Clostridium difficile infection: a systematic review and network meta-analysis. Lancet Infect Dis. 2018;18(9): 1035-44.

25. Ma Y, Yang JY, Peng X, Xiao KY, Xu Q, Wang C. Which probiotic has the best effect on preventing Clostridium difficile-associated diarrhea? A systematic review and network meta-analysis. J Dig Dis. 2020;21(2):69-80.

26. Okumura H, Fukushima A, Taieb V, Shoji S, English M. Fidaxomicin compared with vancomycin and metronidazole for the treatment of Clostridioides (Clostridium) difficile infection: a network metaanalysis. J Infect Chemother. 2020;26(1):43-50.

27. Sridharan K, Sivaramakrishnan G. Which antimicrobial agent is likely to be the best for treating Clostridium difficile infections? A bayesian network meta-analysis of randomized clinical trials. Drug Res. 2019;69(4):194-200. 
28. Hutton B, Salanti G, Caldwell DM, et al. The PRISMA extension statement for reporting of systematic reviews incorporating network meta-analyses of health care interventions: checklist and explanations. Ann Intern Med. 2015;162(11): 777-84.

29. Surawicz CM, Brandt LJ, Binion DG, et al. Guidelines for diagnosis, treatment, and prevention of Clostridium difficile infections. Am J Gastroenterol. 2013;108(4):478-98 (quiz 499).

30. Julian Higgins JT, Chandler J. Cochrane handbook for systematic reviews of interventions version 6.0. Cochrane, 2019. https://training.cochrane.org/ handbook/current. Accessed 29 Aug 2020.

31. Schünemann HBJ, Guyatt G, Oxman A, editors. GRADE handbook for grading quality of evidence and strength of recommendations. Updated October 2013: the GRADE Working Group. https:// training.cochrane.org/handbook/current/chapter14. Accessed 29 Aug 2020.

32. Hvas CL et al. Fecal Microbiota transplantation is superior to fidaxomicin for treatment of recurrent clostridium difficile Infection. Gastroenterology, 2019; 156(5):1324-32.

33. Hota SS, Sales V, Tomlinson G, et al. Oral vancomycin followed by fecal transplantation versus tapering oral vancomycin treatment for recurrent Clostridium difficile infection: an open-label, randomized controlled trial. Clin Infect Dis. 2017;64: 265-71.
34. van Nood E, Vrieze A, Nieuwdorp M, et al. Duodenal infusion of donor feces for recurrent Clostridium difficile. N Engl J Med. 2013;368(5):407-15.

35. Cammarota $G$, et al. Randomised clinical trial: faecal microbiota transplantation by colonoscopy vs. vancomycin for the treatment of recurrent Clostridium difficile infection. Aliment Pharmacol Ther. 2015;41(9):835-43.

36. Johnson $\mathrm{S}$, et al. Vancomycin, metronidazole, or tolevamer for Clostridium difficile infection: results from two multinational, randomized, controlled trials. Clin Infect Dis. 2014;59(3):345-54.

37. Mattila E, et al. A randomized, double-blind study comparing Clostridium difficile immune whey and metronidazole for recurrent Clostridium difficileassociated diarrhoea: efficacy and safety data of a prematurely interrupted trial. Scand J Infect Dis. 2008;40(9):702-8.

38. Haifer C, Kelly CR, Paramsothy S, Andresen D, Papanicolas LE, McKew GL, Borody TJ, Kamm M, Costello SP, Andrews JM, Begun J, Chan HT, Connor S, Ghaly S, Johnson PDR, Lemberg DA, Paramsothy R, Redmond A, Sheorey H, van der Poorten D, Leong RW. Australian consensus statements for the regulation, production and use of faecal microbiota transplantation in clinical practice. Gut. 2020;69(5):801-10.

39. Chang JY, Antonopoulos DA, Kalra A, Tonelli A, Khalife WT, Schmidt TM, Young VB. Decreased diversity of the fecal microbiome in recurrent-associated Diarrhea. J Infect Dis. 2008;197(3):435-8. 